\title{
BMJ Open Electroacupuncture for poststroke spasticity (EAPSS): protocol for a randomised controlled trial
}

\author{
Yiyi Cai, ${ }^{1,2}$ Claire Shuiqing Zhang, ${ }^{2}$ Wenwei Ouyang, ${ }^{1}$ Jianmin $\mathrm{Li},{ }^{1}$ Wenheng Nong, ${ }^{1}$ \\ Anthony Lin Zhang, ${ }^{2}$ Charlie Changli Xue, ${ }^{1,2}$ Zehuai Wen ${ }^{1}$
}

To cite: Cai Y, Zhang CS, Ouyang $\mathrm{W}$, et al.

Electroacupuncture for poststroke spasticity (EAPSS): protocol for a randomised controlled trial. BMJ Open 2018;8:e017912. doi:10.1136/ bmjopen-2017-017912

- Prepublication history and additional material for this paper are available online. To view these files, please visit the journal online (http://dx.doi. org/10.1136/bmjopen-2017017912).

CCX and ZW contributed equally.

Received 25 May 2017

Revised 18 December 2017

Accepted 17 January 2018

Check for updates

${ }^{1}$ Guangdong Provincial Hospital of Chinese Medicine (The Second Affiliated Hospital of Guangzhou University of Chinese Medicine), Guangdong Provincial Academy of Chinese Medical Sciences and The Second Clinical College, Guangzhou University of Chinese Medicine, Guangzhou, Guangdong, China ${ }^{2}$ China-Australia International Research Centre for Chinese Medicine, School of Health and Biomedical Sciences, RMIT University, Melbourne, Victoria, Australia

Correspondence to

Dr Zehuai Wen;

wenzehuai@139.com

\section{ABSTRACT}

Introduction Spasticity is a common complication of stroke. Current therapies for poststroke spasticity (PSS) have been reported to be associated with high costs, lack of long-term benefit and unwanted adverse events (AES). Electroacupuncture (EA) has been used for PSS, however, its efficacy and safety is yet to be confirmed by highquality clinical studies. This study is designed to evaluate the add-on effects and safety profile of EA when used in combination with usual care (UC).

Methods and analysis This study is a parallel group randomised controlled trial. A total of 136 participants will be included and randomly assigned to either the treatment group (EA plus UC) or the control group (UC alone). Prior to the main trial, a pilot study involving 30 participants will be conducted to assess the feasibility of the trial protocol. EA will be administered by registered acupuncturists for $20 \mathrm{~min}$ to $30 \mathrm{~min}$, three times per week for 4 weeks. The primary outcome measure (Modified Ashworth Scale) and secondary outcome measures (Fugl-Meyer Assessment and Barthel Index) will be evaluated at baseline, the end of treatment (week 4) and the end of follow-up (week 8). AEs will be monitored, recorded and reported, and their causality will be explored.

Ethics and dissemination Ethics approval was obtained from the ethics committees of Guangdong Provincial Hospital of Chinese Medicine and RMIT University in December 2016. The results will be disseminated in a peer-reviewed journal, and PhD theses and might be presented at international conferences.

Trial registration number ChiCTR-IOR-16010283; Preresults.

\section{INTRODUCTION}

As one of the most commonly seen complications of stroke, ${ }^{12}$ spasticity can have a negative impact on around $20 \%-50 \%$ of stroke survivors. ${ }^{34}$ The direct cost for patients with poststroke spasticity (PSS) is reported nearly four times higher than those without it. ${ }^{5}$

In clinical management of PSS, treatment options include pharmacological and non-pharmacological interventions. Medications including oral baclofen, botulinum injection and chemical neurolysis may be considered for managing moderate or severe

\section{Strengths and limitations of this study}

- Rigorous randomisation and allocation concealment methods will be applied with outcome assessors and statisticians blinded.

- Treatment effects on spasticity severity, motor function, activities of daily living, as well as adverse events will be observed, recorded and reported.

- Results will be reported complying with Consolidated Standards of Reporting Trials 2010 and the Revised Standards for Reporting Interventions in Controlled Trials of Acupuncture 2010.

- Usual care may vary across participants, potentially limiting the comparability of the two groups.

- Though widely accepted and used, the validity of the Modified Ashworth Scale to measure spasticity has been questioned.

cases of PSS. ${ }^{16-11}$ Unwanted side effects, such as muscle weakness, may limit the use of oral antispasticity medications. ${ }^{12}$ Intrathecal baclofen injection is effective but usually only used in generalised spasticity when there is no response with conservative options. ${ }^{13}$ Non-pharmacological interventions, such as antispastic pattern positioning and rangeof-motion exercises are recommended as primary treatments. ${ }^{16-11}$

Nevertheless, even after various treatments, moderate to severe disability was observed in more than half of patients with spasticity with stroke. ${ }^{14}$ Concerns have been raised about the unsatisfactory long-term benefit and the high cost of current therapies. ${ }^{15} 16$ Due to these limitations of current clinical management for PSS, patients with stroke are in need of alternative treatment options for better control of spasticity with less side effects.

Acupuncture has long been practised in China and in recent decades it has been increasingly popular in the Western countries. $^{2}$ Electroacupuncture (EA) involves the application of an electrical stimulus, where electric current passes between pairs of acupuncture needles. ${ }^{17}$ One advantage of EA 
over manual acupuncture is that the frequency and intensity can be standardised and repeated easily, ensuring participants in clinical studies receive the same amount of stimulation. ${ }^{7}$ Besides, EA was found to be more effective than manual acupuncture in a previous systematic review evaluating acupuncture for PSS. ${ }^{2}$ However, methodological issues and inadequate reporting of included studies limits the certainty of these findings. ${ }^{2}$

Prior to designing this clinical trial, we had conducted a systematic review of EA for PSS (International prospective register of systematic reviews (PROSPERO) 2015: CRD42015027894). ${ }^{18}$ Twenty-two randomised controlled trials were included in the meta-analysis. The following limitations prevented us from drawing a firm conclusion on the effects and safety of EA for PSS: (1) methodological quality: none of the included trials applied appropriate allocation concealment, and only three studies implemented blinding of outcome assessors; (2) reporting quality: none of the included studies addressed all key items recommended by the Standards for Reporting Intervention in Controlled Trials of Acupuncture 2010 $\left(\right.$ STRICTA 2010) ${ }^{19}$; 3) absence of safety data: none of the included trials evaluate the safety of EA in the treatment for PSS.

This study aims to evaluate the effects and safety profile of EA for PSS and address the abovementioned limitations. The main hypothesis of this trial is that, compared with usual care (UC, usual pharmacotherapy and/or rehabilitation therapies) alone, adding EA to UC will have an additional benefit on PSS in terms of muscle tone reduction, improvement of motor function of affected extremities and activities of daily living. The results of this trial will be reported in accordance with the Consolidated Standards of Reporting Trials (CONSORT) ${ }^{20} 2010$ and STRICTA. ${ }^{19}$ It was designed following the instructions of the Standard Protocol Items: Recommendations for Interventional Trials (SPIRIT) 2013 checklist (online supplementary appendix 1 ). ${ }^{21}$ Appropriate allocation concealment and blinding of outcome assessors/ statisticians will be adopted in the design of this trial, with evaluation of the effects and safety of EA in a 4-week treatment phase and a 4-week follow-up phase.

\section{METHODS AND ANALYSIS \\ Study design}

This study will be a single-centre, outcome-assessor-blinded parallel randomised controlled trial consisting of a 4-week treatment phase followed by a 4-week follow-up phase. Figure 1 shows the trial procedure and table 1 details the trial schedule.

\section{Setting, recruitment and participants}

This trial will be conducted at the Guangdong Provincial Hospital of Chinese Medicine (GPHCM) in Guangzhou, Guangdong Province, China. Potential participants will be recruited through advertising posters from the inpatient and outpatient department of rehabilitation, department of acupuncture as well as the department of neurology of the hospital. Patients may also be referred to the trial by their doctors (general practitioners, neurologists, physiotherapists or acupuncturists). The recruiting criteria are as below:

\section{Inclusion criteria}

The inclusion criteria are: (1) people with first-ever stroke confirmed by CT or/and MRI; (2) 30 days to 1 year after stroke onset; (3) aged $\geq 18$ years; (4) baseline Modified Ashworth Scale (MAS) $\geq 1$; (5) willing to participate and be randomised to one of the groups.

\section{Exclusion criteria}

The exclusion criteria are: (1) people with spasticity due to other causes, such as multiple sclerosis, traumatic brain and spinal cord injuries; (2) severe and unstable clinical disorders; (3) unable to follow or respond to treatment instructions; (4) lactation, pregnancy or intend to be pregnant within 6 months; (5) pacemaker or implantable cardioverter defibrillator carriers; (6) currently enrolled in another clinical trial; (7) needle phobia.

Written informed consent will be sought from participants once their eligibility is confirmed. Written information and verbal explanation of the study (including the purpose, procedures, time commitment, potential risks and benefits of the trial) will be provided before written consent is obtained.

All participants will receive a unique research identification number, which will be the only identifier on data collection forms and in the electronic database. Electronic data will be kept confidential and stored in password-protected electronic files, and all hard-copy files will be stored in locked filing cabinets. Compensation for any harm related to the trial will be provided by GPHCM and will be made on a case-by-case basis.

Participants are permitted to withdraw at any time during the trial, with or without providing a reason. All withdrawn cases who have received the intervention will be contacted 4 weeks after withdrawal as a follow-up assessment, to obtain information about their condition.

\section{Randomisation and allocation concealment}

Participants will be randomly allocated to an intervention group (EA plus UC) or control group (UC alone) at a ratio of $1: 1$. An external statistician will provide the computer-generated random sequences, and implement allocation by a central web-based interactive randomisation service system at GPHCM. Block randomisation will be used to ensure equal numbers of participants are allocated to the two groups. Participants will be advised not to reveal their group allocation to the outcome assessors at any time during the trial.

\section{Blinding}

Due to the difficulty of blinding participants and acupuncturists in trials with EA, this study was designed as an open-label trial. Independent outcome assessors and 


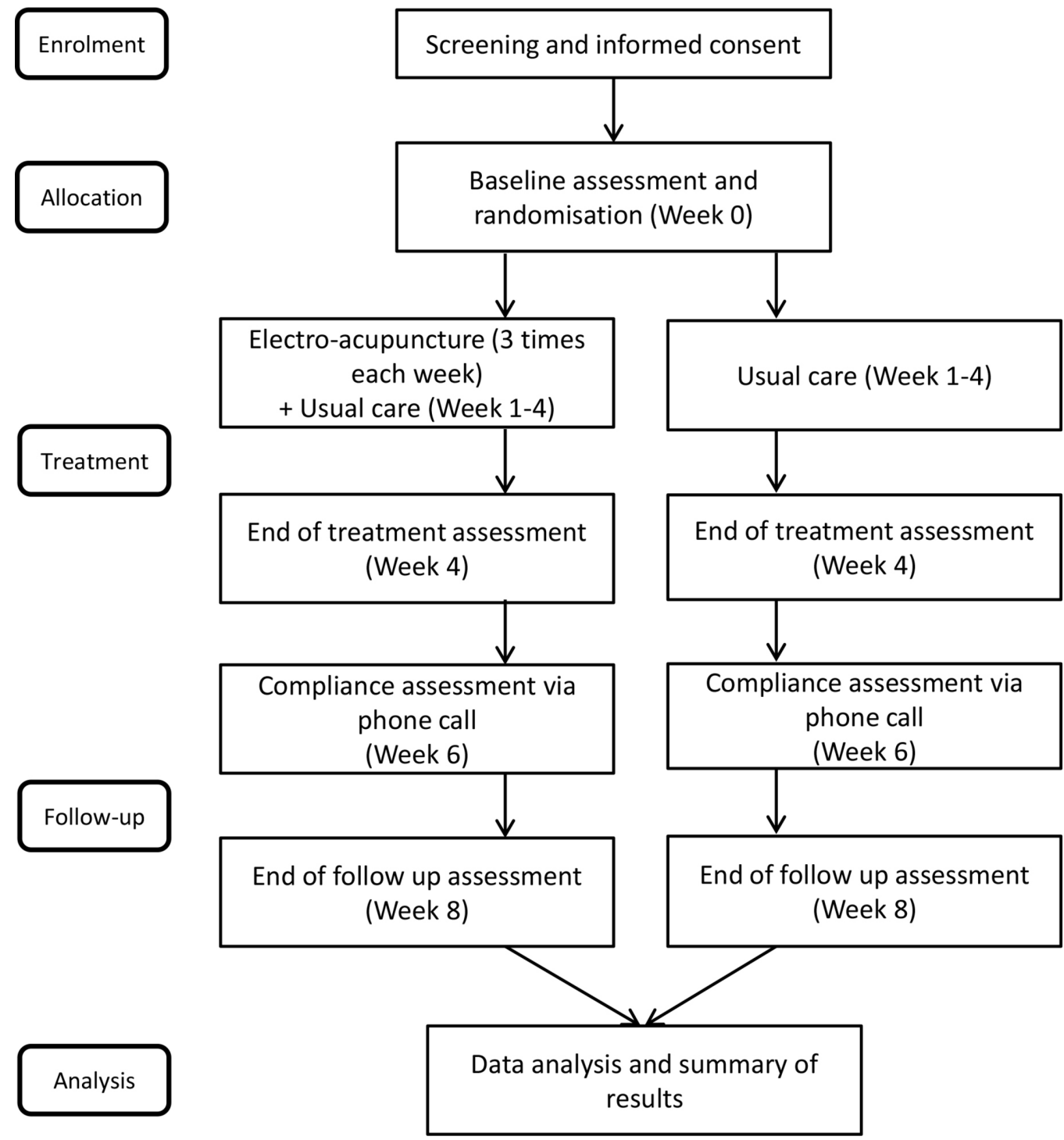

Figure 1 Flow chart.

statisticians will be blinded to group allocation throughout the entire trial.

\section{Interventions}

In the 4-week treatment phase, participants in both groups will receive $\mathrm{UC}$, and those allocated to the intervention group will also receive EA treatments.

\section{UC treatment in both groups}

Considering that recommendations for management of PSS vary across different clinical guidelines, and that patients with stroke may have complex comorbidities and complications, the UC therapies used in this trial will not be strictly standardised. UC for spasticity which is tailored for the individual may include pharmacological and non-pharmacological interventions. Treatments of general internal medicine (such as blood pressure control, lipid-lowering medications) and rehabilitation therapies will be permitted. Details of UC used during the trial will be recorded, noting any change of $\mathrm{UC}$ and the reasons.
EA add-on treatment in the intervention group

A total of 12 EA sessions, 3 per week, will be performed for a period of 4 weeks. Acupuncturists who deliver the treatments are qualified traditional Chinese medicine practitioners with at least 2 years of acupuncture experience. They are required to be registered at the Chinese Medical Doctor Association and hold a master's degree in acupuncture. All EA treatment will be conducted in private rooms. The practitioner will explain the EA process and possible sensations during EA therapies to the participants before treatment. Immediately after each EA session, brief interviews will be conducted to record participants' feedback about the treatment. These forms will be checked regularly by the investigators for consistency across different acupuncture therapists.

In the EA treatment, needling may be bilateral or unilateral, at the acupuncturist's discretion. Based on our systematic review of published EA trials for PSS, ${ }^{18}$ a list of acupuncture points were identified and suggested to be used in this trial: LI4, LI10, LI11, LI15, TE5, GB34, 
Table 1 Schedule of enrolment, interventions and assessments

\begin{tabular}{|c|c|c|c|c|c|c|}
\hline \multirow[b]{2}{*}{ Time point } & \multirow{2}{*}{$\begin{array}{l}\text { Screening } \\
\text { Enrolment }\end{array}$} & \multirow{2}{*}{$\begin{array}{l}\text { Baseline } \\
\text { assessment } \\
\text { Week } 0\end{array}$} & \multicolumn{2}{|c|}{ Treatment phase } & \multicolumn{2}{|c|}{ Follow-up phase } \\
\hline & & & Weeks 1-3 & Week 4 & Week 6 & Week 8 \\
\hline Eligibility screening & $x$ & & & & & \\
\hline Informed consent & $x$ & & & & & \\
\hline Demographic characteristics & & $x$ & & & & \\
\hline $\begin{array}{l}\text { History and treatment of spasticity and } \\
\text { comorbidities }\end{array}$ & & $x$ & & & & \\
\hline MAS & & $x$ & & $x$ & & $x$ \\
\hline FMA & & $x$ & & $x$ & & $x$ \\
\hline $\mathrm{BI}$ & & $x$ & & $x$ & & $x$ \\
\hline EA therapy & & & $x$ & $x$ & & \\
\hline EA treatment record & & & $x$ & $x$ & & \\
\hline UC (both groups) & & & $x$ & $x$ & $x$ & $x$ \\
\hline AEs & & & $x$ & $x$ & $x$ & $x$ \\
\hline Dropouts & & & $x$ & $x$ & $x$ & $x$ \\
\hline Record of clinic or hospital visits & & & $x$ & $x$ & $x$ & $x$ \\
\hline
\end{tabular}

AEs, adverse events; BI, Barthel Index; EA, electroacupuncture; FMA, Fugl-Meyer Assessment; MAS, Modified Ashworth Scale; UC, usual care; $x$, items required.

LR3, SP6, ST36 and ST40. ${ }^{22}$ Details of these points were available in online supplementary table $\mathrm{S} 1$. The selection of acupuncture points may depend on the treatment needed for the joints affected by spasticity, with LI4, TE5 (wrist), LI10, LI11 (elbow), LI15 (shoulder), GB34, ST36, ST40 (knee), SP36 (ankle) and LR3 (toe joint) as mandatory points. In addition, acupuncturists will be permitted to select other points rather than those included in this list as optional points based on the individualised condition of patients. It is important to note that all acupuncturists will be required to select points located in the antagonistic muscles and avoid those located in the spasticity muscles. A maximum of 10 needles, including a maximum of 3 pairs for EA on the affected side, will be inserted in each treatment session. ${ }^{23}$ Details of each EA treatment session including the acupuncture points will be documented.

Single-use stainless steel acupuncture needles of $0.25 \mathrm{~mm}$ diameter (HanyiTCM, Beijing, China) will be used for EA treatments. Needle lengths may vary from $25 \mathrm{~mm}$ to $40 \mathrm{~mm}$ with needle insertion depth varying from $15 \mathrm{~mm}$ to $25 \mathrm{~mm}$ according to the thickness of muscles where the EA will be conducted. 'De qi' sensation, an elicited response by patients, will be obtained before adding electric stimulation. 'De qi' is a composite of unique needling sensations, including aching, numbness, distension or heaviness felt by both patients and acupuncturists. ${ }^{24}$ On obtaining the needling sensation, EA will be applied through a stimulator (G6805-I, Qing Dao Xinshen Industrial, Qingdao, China) with biphasic continuous waves at a frequency of $50-100 \mathrm{~Hz}$ and at the maximum tolerable intensity for 20-30 min.

\section{Follow-ups}

Participants in both groups will continue to receive UC during the 4-week follow-up phase. Participants will be contacted by phone at the end of week 6 for compliance assessment. At the end of the follow-up phase, participants will attend the trial clinic for a final assessment, then participants' involvement in the trial will cease.

\section{Outcome measures and evaluation}

The primary outcome measure is the changes in MAS (online supplementary appendix 2) score of the major affected joint from baseline to the end of treatment. MAS measures the resistance to passive movement on a 6-point scale (levels $0,1,1+, 2,3$ and 4 ).$^{25}$ A lower score indicates a smaller resistance in the measured joint. MAS in six different joints (shoulder, elbow, wrist, hip, knee and ankle) in the hemiplegia side will be measured and recorded at baseline (week 0), end of treatment (week 4) and end of follow-up (week 8).

The secondary outcome measures are the Fugl-Meyer Assessment of motor performance (FMA (motor function)) (online supplementary appendix 3), and Barthel Index (BI) (online supplementary appendix 4). FMA (motor function) was developed from the FMA of sensorimotor recovery. The FMA (motor function) sums the scores for the upper limb (maximum score of 66) and the lower limb (maximum score of 34) to obtain a total score (maximum 100). ${ }^{26} \mathrm{~A}$ higher result in FMA suggests a better recovery in motor function. BI (online supplementary appendix 4) is a 10-item instrument that evaluates activities of daily living. Items include feeding, bathing, grooming, dressing, bowels, bladder, toilet use, transfers 
(bed to chair and back), mobility (on level surface) and stairs. ${ }^{27} \mathrm{BI}$ establishes the degree of independence from physical assistance for important tasks. Scores range from 0 to 100 , with a higher score meaning less dependent to help.

Baseline data will be collected at the first visit by an independent outcome assessor, including participant's family history, medical history and presenting symptoms, potential risk factors and aggravating factors for spasticity, complications and comorbidities, as well as evaluation of MAS, FMA and BI (table 1). At the end of the treatment and follow-up phases, MAS, FMA and BI will be evaluated by the same outcome assessor.

\section{Safety assessment}

All participants will be requested to report information of any adverse event (AE) occurring during the trail. All details of AEs including the time of occurrence and severity will be recorded. The relationship between AEs and the intervention will be assessed according to the WHO Uppsala Monitoring Centre System for Standardised Case Causality Assessment. ${ }^{28}$ EA is widely accepted as relatively safe if applied appropriately by competent practitioners. $^{29}$ If participants develop an $\mathrm{AE}$ during the trial, they will be advised to seek medical advice from their physicians and cease the EA treatment when the $\mathrm{AE}$ is considered to be EA-related and becomes intolerable. Severe AEs (SAEs) or severe adverse reactions (SARs) are defined according to the International Conference on Harmonisation harmonised tripartite guideline $^{30}$ and must be reported to the ethics committee of GPHCM and the research team.

\section{Discontinuing interventions}

The determination to discontinue the trial for a participant will be made by the research team if: (1) a serious poststroke complication develops; or (2) recurrent stroke or any other severe condition occurs leaving the patient in a critical condition. Physicians will determine whether the participants should cease the trial participation in the event of an SAE or SAR.

\section{Data collection, management and monitoring}

Information of the participants, instrument measures and other relevant data will be collected at the baseline, post-treatment and follow-up assessments. AEs will be observed and recorded during the 8-week duration of the trial. The case report form (CRF) including EA treatment records will be paper-based. All corrections made to the CRFs must be personally signed and dated by the person responsible. All data will then be entered into a predesigned, password-protected electronic data set by two independent investigators who are blinded to group allocation. Double-checking of entered data will be performed by another researcher to ensure accuracy. The original CRFs and all other forms will be kept securely in GPHCM. Only members of the trial research team will have access to the final trial data set.
Data and progress monitoring will be conducted by an independent researcher outside the research team every 2 weeks throughout the trial. The independent data management team will monitor the safety and implementation of the trial, and will have access to group allocation.

\section{Sample size calculation}

A pilot study of 30 participants will be conducted to test the feasibility of the trial protocol prior to a full-scale trial. Sample size estimation for the full-scale trial is based on a meta-analysis of six trials reporting MAS. ${ }^{29}{ }^{31-35}$ The assumption is that adding $\mathrm{EA}$ to $\mathrm{UC}$ is more effective than $\mathrm{UC}$ alone. Accordingly, the EA group and the UC group would show a decrease of 1.22 and 0.79 in MAS scores, respectively, with an SD of 0.72 in each group. Sample size was estimated using software power analysis and sample size V.11.0 (NCSS, Kaysville, Utah, USA), with a significance level $(\alpha=0.05)$ of a two-sided two-sample t-test and $80 \%$ power to detect a difference between the two groups. A total sample size of 136 will be required, allowing for equal allocation to the two groups and $15 \%$ loss of follow-ups. On completion of the pilot study, results of the main outcomes will be used to adjust the sample size estimation for the full-scale trial.

\section{Statistical analysis}

The Statistical Package for the Social Sciences software V.20.0 for Windows (IBM SPSS Statistics, IBM Corp, Somers, New York, USA) and SAS V.9.2 (SAS Institute, Cary, North Carolina, USA) will be used for data analysis. For all analyses, the statistical significance levels were set at $5 \%(a=0.05)$.

Demographic and other baseline characteristics will be summarised for each group. Categorical variables will be presented using frequencies and percentages, and continuous variables using standard measures of central tendency and dispersion with mean and SD if data are normally distributed. Data not normally distributed will be presented using median and IQR will be used.

To compare the changes of primary and secondary outcomes (MAS, FMA and BI) between groups, a superiority independent sample t-test with a $95 \%$ CI will be conducted. Further, repeated measures analysis of variance or a liner mixed model will be applied to analyse the changes of MAS, FMA and BI across three testing time points (weeks 0,4 and 8). AEs will be expressed by tabulations and presented with descriptive statistics. Frequencies and percentages for AEs will also be analysed using $\chi^{2}$ test or Fisher's exact test to detect between-group differences.

Efficacy analyses will be performed using an intentto-treat approach. Missing data will be replaced by the multiple imputation method. Ten imputed data sets will be created under the assumption that missing data are random. Result of the trial will then be the pooled intervention effects based on the analyses of the data sets with multiple imputations. 
Sensitivity analyses will be performed to assess the unimputation and complete case analyses. In addition, to adjust for hypothesised residual baseline imbalances and to assess their impact on effect estimates, a multivariable analysis will be performed. Moreover, subgroup analysis of muscle tone measured by MAS will be conducted with regard to age ( $<65$ years and $\geq 65$ years), gender, different types of stroke, baseline MAS, affected joints and length of the poststroke period to specify effects of EA in these domains.

\section{ETHICS AND DISSEMINATION}

The trial protocol has been approved by the Ethics Committee of GPHCM (online supplementary appendix $5,6)$ and the Royal Melbourne Institute of Technology (RMIT) University Human Research Ethics Committee (online supplementary appendix 7) in December 2016 in accordance with the related Chinese ethics audit regulations and the Australian National Health and Medical Research Council's National Statement of Ethical Conduct in Human Research. ${ }^{36-38}$ Any modification in protocol is not expected unless necessary. Any change in the selection criteria or methodology will be discussed with the entire research team and approved by the ethics committees. The results will be published in a peer-reviewed journal, a $\mathrm{PhD}$ thesis and presented at conferences. Data will be published in aggregate to avoid individual participant identification and presented in such a way that identifiable data are removed.

\section{DISCUSSION}

\section{Summary of the trial}

Based on the results of synthesis results of a systematic review, ${ }^{18}$ this trial was designed to evaluate the effects and safety of EA plus UC for PSS with a semipragmatic EA intervention protocol. Evaluation of MAS, FMA and $\mathrm{BI}$, as well as AEs, will be recorded and reported in a 4-week treatment and 4-week follow-up period. Moreover, this study will incorporate appropriate allocation concealment and blinding of assessors/statisticians and will be reported in accordance with CONSORT 2010 and STRICTA 2010. ${ }^{19} 20$

\section{EA treatment protocol}

EA treatment protocol, including the electrical stimulation frequency, optional acupuncture points, number, frequency and duration of treatment, was designed based on the results of a systematic review. ${ }^{18}$ It is worth noting that although there is no consensus on the selection of acupuncture points for EA in PSS, it had been reported that electrical stimulation might induce muscle contraction. ${ }^{39}$ Acupuncture practitioners will be required to avoid stimulating acupuncture points located in the spasm muscles, and the maximum number of acupuncture points for EA on the affected side of the body will be limited to three pairs. ${ }^{23}$ Selection of appropriate electrical stimulation frequency was one of the major challenges in the study design. We referred to the results of our systematic review ${ }^{18}$ and selected a biphasic continuous waveform $(50-100 \mathrm{~Hz})$ at patients' maximum tolerable intensity for the following reasons: first, there is no specific instruction or consensus for electrical stimulation frequency and waveform from existing publications that could guide EA parameters' selection for spasticity; second, it was reported that low electrical stimulation frequency is likely to induce visible muscle contraction; ${ }^{39}$ third, all published randomised controlled trials included in the meta-analysis $^{18}$ used biphasic continuous waveforms. After discussion with acupuncturists in GPHCM, where the trial will be conducted, we decided to use biphasic continuous waveforms $(50-100 \mathrm{~Hz})$ in this trial.

\section{Control method for EA trial}

In order to achieve a more pragmatic control method, UC including pharmacotherapy and rehabilitation treatment, will not be strictly standardised. Pseudoacupuncture, sham acupuncture and placebo acupuncture were considered an inappropriate choice for control for several reasons. Positive treatment effects of pseudoacupuncture and sham acupuncture with needle insertion have been observed. ${ }^{40-42}$ In this scenario, treatment effects of acupuncture might be underestimated when compared with pseudoacupuncture or sham acupuncture. Further, this trial will recruit patients with stroke from a hospital in mainland China, where EA is well accepted and widely used. Patients are generally familiar with EA, making participant's blinding difficult. ${ }^{2}$

\section{Selection of outcome measures}

Due to the complex relationship between poststroke pathological lesions and various complications, it is difficult to attribute changes in impairment, function and activity after stroke to spasticity alone. ${ }^{43}$ Spasticity can impact on patients with stroke in various ways. ${ }^{15}$ Therefore, the assessment of patients with stroke with spasticity should not be limited to the severity of spasticity alone, but should include a comprehensive measurement of motor function and activity of daily living in general.

MAS in the upper and lower extremities demonstrated adequate to excellent test-retest reliability among patients with stroke. ${ }^{44}$ In terms of inter-rater reliability, high agreement was detected with MAS when assessment was made by a single rater, but there was poor reliability between different examiners, particularly for MAS in the lower limb. ${ }^{44}$ With regards to validity, MAS has been criticised for producing a global assessment of the resistance to passive movement of an extremity rather than a specific assessment of stretch-reflex hyperexcitability. ${ }^{45}$ While the ability of the MAS to distinguish between functional and mechanical properties of muscle has been questioned, ${ }^{47}$ it is considered as the primary measure of muscle spasticity with widespread clinical acceptance. In fact, MAS is considered the current clinical gold standard, recommended by the American Physical Therapy Association, 
in the the absence of objective clinical tools for measuring spasticity that are clinically feasible and easily interpreted by clinicians. ${ }^{48}{ }^{49}$ Although there has not been an officially translated Chinese version of MAS and FMA yet, the outcome assessor of our trial is a professional rehabilitation therapist with many years' experience of using MAS and FMA in clinical practice, so we consider the use of these outcome measures acceptable in this trial.

FMA is one of the most widely accepted and recommended quantitative assessment tools to evaluate the recovery of patients with stroke in both clinical and research settings. FMA has excellent performance in reliability and validity ${ }^{50}$ in terms of motor function, although criticism has been made of the representation of distal fine motor function and finger movement. ${ }^{51} \mathrm{BI}$ was originally published in 1965 and has been widely accepted, used and studied with adequate to excellent validity and reliability. ${ }^{52-54}$ More recently, a new outcome measure, the Functional Independence Measure (FIM), was developed to replace BI for accessing activities of daily living. ${ }^{55}$ Uptake of this new assessment tool in trials involving Chinese people with PSS is low. Given that BI has been widely accepted, used, studied and reported in $\mathrm{China}^{52}$ and that the psychometric properties of the FIM instrument and BI are similar, ${ }^{486}$ BI has been selected for this trial. This will allow for comparison of our results with other studies.

The results of the study could provide evidence of high methodological and reporting quality on the effects and safety of EA for PSS. Specifically, muscle tone, motor function, activities of daily living and adverse events will be evaluated and analysed.

\section{TRIAL STATUS}

Patient recruitment began in March 2017 and is expected to finish at the end of 2019.

Acknowledgements The authors thank all involved in the study, the team members, therapists, assessors and participants. The authors also thank all the patients and their caregivers who participated in this study.

Contributors YC, CSZ, ALZ and ZW designed or conceptualised the trial protocol, and drafted and revised the manuscript. WO checked the statistical analysis plan. CSZ, ALZ and CCX revised the manuscript. YC, JL and WN will conduct the trial. All the authors have read, revised and approved this version of the manuscript.

Funding This trial is supported by the 'Construction of High-level University' Public Projects from Guangzhou University of Chinese Medicine (No. (2016) 64), and the 'Specific Research Fund for TCM Science and Technology of Guangdong Provincial Hospital of Chinese Medicine (2016)' (No. YN2016QL01) and the 'National Key Technology R\&D Program for the 12th Five-year Plan of Ministry of Science and Technology, China' (No. 2013BAl02B10).

Competing interests None declared.

Patient consent Not required.

Ethics approval The ethics Committees of Guangdong Provincial Hospital of Chinese Medicine and RMIT University.

Provenance and peer review Not commissioned; externally peer reviewed.

Data sharing statement The results will be published in a peer-reviewed journal, a PhD thesis and presented at conferences. Data will be published in group form to avoid individual participant identification and presented in such a way that identifiable data is removed.
Open Access This is an Open Access article distributed in accordance with the Creative Commons Attribution Non Commercial (CC BY-NC 4.0) license, which permits others to distribute, remix, adapt, build upon this work non-commercially, and license their derivative works on different terms, provided the original work is properly cited and the use is non-commercial. See: http://creativecommons.org/ licenses/by-nc/4.0/

(c) Article author(s) (or their employer(s) unless otherwise stated in the text of the article) 2018. All rights reserved. No commercial use is permitted unless otherwise expressly granted.

\section{REFERENCES}

1. Miller EL, Murray L, Richards L, et al. Comprehensive overview of nursing and interdisciplinary rehabilitation care of the stroke patient: a scientific statement from the American Heart Association. Stroke 2010;41:2402-48.

2. Lim SM, Yoo J, Lee E, et al. Acupuncture for spasticity after stroke: a systematic review and meta-analysis of randomized controlled trials. Evid Based Complement Alternat Med 2015;2015:1-12.

3. Zorowitz RD, Gillard PJ, Brainin M. Poststroke spasticity: sequelae and burden on stroke survivors and caregivers. Neurology 2013;80(Suppl 2):S45-52.

4. Kwah LK, Harvey LA, Diong JH, et al. Half of the adults who present to hospital with stroke develop at least one contracture within six months: an observational study. J Physiother 2012;58:41-7.

5. Lundström E, Smits A, Borg J, et al. Four-fold increase in direct costs of stroke survivors with spasticity compared with stroke survivors without spasticity: the first year after the event. Stroke 2010;41:319-24.

6. National Stroke Foundation. Clinical Guidelines for Stroke Management 2010. Melbourne, Australia. https://informme.org. $\mathrm{au} /$ guidelines/clinical-guidelines-for-stroke-management-2010 (accessed on 7 Jun 2017).

7. Smith LN, James R, Barber M, et al. Rehabilitation of patients with stroke: summary of SIGN guidance. BMJ 2010;340:c2845.

8. Stroke Foundation of New Zealand and New Zealand Guidelines Group. Clinical Guidelines for Stroke Management 2010. Wellington: Stroke Foundation of New Zealand, 2010. (accessed 7 Jun 2017).

9. Chinese Society of Neurology and Stroke Prevention Project Committee of National Health and Family Planning Commission in China. China post-stroke rehabilitation guideline [Chinese]. Chinese Journal of Rehabilitation Theory and Practice 2012;18:18.

10. National Institute for Health and Care Excellence (NICE). Stroke rehabilitation in adults clinical guideline [CG162]. 2013 https://www. nice.org.uk/guidance/cg162 (accessed 24 Jan 2017).

11. Lindsay P, Furie KL, Davis SM, et al. World Stroke Organization global stroke services guidelines and action plan. Int J Stroke 2014;9(Suppl A100):4-13.

12. Fan LB, Liu SZ, Wang ZT. Application of Electro-acupuncture plus Movement Therapy in Recovering Neurologic Function of Patients with Spastic Hemiplegia. Shanghai Journal of Acupuncture and Moxibustion 2015;34:1.

13. Harned ME, Salles SS, Grider JS. An introduction to trialing intrathecal baclofen in patients with hemiparetic spasticity: a description of 3 cases. Pain Physician 2011;14:483-9.

14. Sze $\mathrm{KH}$, Wong $\mathrm{E}$, Or $\mathrm{KH}$, et al. Factors predicting stroke disability at discharge: a study of 793 Chinese. Arch Phys Med Rehabil 2000;81:876-80.

15. Nair KP, Marsden J. The management of spasticity in adults. BMJ 2014;349:g4737.

16. Wu P, Mills E, Moher D, et al. Acupuncture in poststroke rehabilitation: a systematic review and meta-analysis of randomized trials. Stroke 2010;41:e171-e179.

17. Liu AJ, Li JH, Li HQ, et al. Electroacupuncture for acute ischemic stroke: a meta-analysis of randomized controlled trials. Am J Chin Med 2015;43:1541-66.

18. Cai Y, Zhang CS, Liu S, et al. Electroacupuncture for Poststroke Spasticity: A Systematic Review and Meta-Analysis. Arch Phys Med Rehabil 2017;98:2578-89.

19. MacPherson H, Altman DG, Hammerschlag R, et al. Revised STandards for Reporting Interventions in Clinical Trials of Acupuncture (STRICTA): extending the CONSORT statement. PLoS Med 2010;7:e1000261.

20. Schulz KF, Altman DG, Moher D. CONSORT 2010 statement: updated guidelines for reporting parallel group randomised trials. BMC Med 2010;8:18. 
21. Chan AW, Tetzlaff JM, Altman DG, et al. SPIRIT 2013 statement: defining standard protocol items for clinical trials. Ann Intern Med 2013;158:200-7.

22. WHO Regional Office for the Western Pacific. WHO Standard Acupuncture Point Locations in the Western Pacific Region. Manila: World Health Oraganization, 2008. http://www.wpro.who.int/ publications/docs/WHOIST_26JUNE_FINAL.pdf (accessed 24 Jan 2017).

23. Wang YY, Xie YM. Practical stroke neurorehabilitation (实用中风病 康复学. Beijing, China: People's Medical Publishing House [Book in Chinese], 2010.

24. Yang $X Y$, Shi GX, Li QQ, et al. Characterization of deqi sensation and acupuncture effect. Evid Based Complement Alternat Med 2013;2013:1-7

25. Bohannon RW, Smith MB. Interrater reliability of a modified Ashworth scale of muscle spasticity. Phys Ther 1987;67:206-7.

26. Fugl-Meyer AR, Jääskö L, Leyman I, et al. The post-stroke hemiplegic patient. 1. a method for evaluation of physical performance. Scand J Rehabil Med 1975;7:13-31.

27. Mahoney FI, Barthel DW. Functional evaluation: the barthel index. Md State Med J 1965;14:61-5.

28. Uppsala Monitoring Centre. The use of the WHO-UMC system for standardised case causality assessment. http://www.who.int/ medicines/areas/quality_safety/safety_efficacy/WHOcausality assessment.pdf (accessed 24 Jan 2017).

29. Liu M, Li ZH, Ma H. Clinical evaluation of electric acupuncture at antagonistic muscle acupoints combined with rehabilitation training for the treatment of apoplexy spastic paralysis. Journal of Clinical Acupuncture and Moxibustion 2016:32:3

30. International Conference on Harmonisation of Techinical Requirements for Registration of Pharmaceuticals for Human Use. $\mathrm{ICH}$ harmonised tripartite guideline: safety pharmacology studies for human pharmaceuticals S7A. http://www.ich.org/fileadmin/ Public_Web_Site/ICH_Products/Guidelines/Safety/S7A/Step4/S7A Guideline.pdf (accessed 24 Jan2017).

31. Lu F. The combination of acupuncture Bobath therapy tendon junction of spastic paralysis after stroke clinical efficacy. Harbin Heilongjiang Province: Heilongjiang University of Chinese Medicine, 2011. [Article in Chinese].

32. Wu W. The effects of acupuncture combined with rehabilitation therapies for strephenopodia in hemiplegia side after stroke. Shanghai Journal of Acupuncture and Moxibustion [Article in Chinese] 2011;30:3.

33. Xue Q, Li S, Huo G. The effects of acupuncture therapy to balance muscle tones for hemiplegia patients with spasticity in motor function rehabilitation. Jiangsu Journal of Traditional Chinese Medicine [Article in Chinese] 2008;40:2.

34. Yu M, Zhang $\mathrm{H}$. The effects of electro-acupuncture combined with rehabilitation exercises for spasticity in hemiplegia patients. Chinese General Practice [Article in Chinese] 2007:10:2.

35. Zhang S, Lei M, Xiao Y, et al. A clinical research on the effects of electro-acupuncture combined with bobath therapy for post-stroke spasticity. Chinese Journal of Public Health [Article in Chinese] 2015;31:2.

36. National Health and Family Planning Commission of the People's Republic of China (中国国家卫生和计划生育委员会令). Measures for the Ethical Review of Biomedical Research Involving Human Subjects (涉及人的生物医学研究伦理审查办法) [Article in Chinese]. No. [2016]11.

37. State Administration of Traditional Chinese Medicine of the People's Republic of China (中国国家中医药管理局). Construction specifications of platform construction assessment of ethics review of traditional chinese medicine (中医药临床研究伦理审查平台建设规 范) [Article in Chinese]. No. [2011]34.

38. The National Health and Medical Research CouncilThe Australian Research CouncilThe Australian Vice-Chancellors' Committee. National statement on ethical conduct in human research 2007 (Updated May 2015). Canberra: Commonwealth of Australia. (accessed 2 Aug 2017).

39. Andersson S, Lundeberg T. Acupuncture--from empiricism to science: functional background to acupuncture effects in pain and disease. Med Hypotheses 1995;45:271-81.

40. Koog YH. Effect of placebo acupuncture over no-treatment: a simple model incorporating the placebo and nocebo effects. Complement Ther Med 2016;24:69-72.

41. Wong EL, Leung PC, Zhang L. Placebo acupuncture in an acupuncture clinical trial. How good is the blinding effect? J Acupunct Meridian Stud 2015;8:40-3.

42. Zhu D, Gao Y, Chang J, et al. Placebo acupuncture devices: considerations for acupuncture research. Evid Based Complement Alternat Med 2013;2013:1-9.

43. Johnson GR. Outcome measures of spasticity. Eur J Neurol 2002;9(Suppl 1):10-16. dicussion 53-61.

44. Blackburn M, van Vliet P, Mockett SP. Reliability of measurements obtained with the modified ashworth scale in the lower extremities of people with stroke. Phys Ther 2002;82:25-34.

45. Kamper DG, Schmit BD, Rymer WZ. Effect of muscle biomechanics on the quantification of spasticity. Ann Biomed Eng 2001;29:1122-34.

46. Scholtes VA, Becher JG, Beelen A, et al. Clinical assessment of spasticity in children with cerebral palsy: a critical review of available instruments. Dev Med Child Neurol 2006;48:64-73.

47. van Wijck FM, Pandyan AD, Johnson GR, et al. Assessing motor deficits in neurological rehabilitation: patterns of instrument usage. Neurorehabil Neural Repair 2001;15:23-30.

48. Neurology Section of the American Physical Therapy Association. Neurology section of StrokEDGE taskforce. Secondary Neurology Section of StrokEDGE Taskforce. $2011 \mathrm{http} / / / \mathrm{www}$.neuropt.org/ professional-resources/neurology-section-outcome-measuresrecommendations/stroke

49. Aloraini SM, Gäverth J, Yeung E, et al. Assessment of spasticity after stroke using clinical measures: a systematic review. Disabil Rehabil 2015;37:2313-23.

50. Page SJ, Hade E, Persch A. Psychometrics of the wrist stability and hand mobility subscales of the Fugl-Meyer assessment in moderately impaired stroke. Phys Ther 2015;95:103-8.

51. Gladstone DJ, Danells CJ, Black SE. The fugl-meyer assessment of motor recovery after stroke: a critical review of its measurement properties. Neurorehabil Neural Repair 2002;16:232-40.

52. Leung SO, Chan CC, Shah S. Development of a Chinese version of the modified barthel index-- validity and reliability. Clin Rehabil 2007;21:912-22.

53. Lin JH, Hsu MJ, Hsu HW, et al. Psychometric comparisons of 3 functional ambulation measures for patients with stroke. Stroke 2010;41:2021-5.

54. Rollnik JD. The Early Rehabilitation Barthel Index (ERBI). Rehabilitation 2011:50:408-11.

55. Dromerick AW, Edwards DF, Diringer MN. Sensitivity to changes in disability after stroke: a comparison of four scales useful in clinical trials. J Rehabil Res Dev 2003;40:1-8.

56. Hsueh IP, Lin JH, Jeng JS, et al. Comparison of the psychometric characteristics of the functional independence measure, 5 item Barthel index, and 10 item Barthel index in patients with stroke. $J$ Neurol Neurosurg Psychiatry 2002;73:188-90. 